2009, 113, 7973-7976

Published on Web 05/18/2009

\title{
Evolution from Surface-Influenced to Bulk-Like Dynamics in Nanoscopically Confined Water
}

\author{
Santiago Romero-Vargas Castrillón, ${ }^{\dagger}$ Nicolás Giovambattista, ${ }^{\dagger}$ Ilhan A. Aksay, ${ }^{\dagger}$ and \\ Pablo G. Debenedetti ${ }^{*, \dagger}$ \\ Department of Chemical Engineering, Princeton University, Princeton New Jersey 08544-5263, and \\ Physics Department, Brooklyn College of the City University of New York, Brooklyn, New York 11210
}

Received: March 20, 2009; Revised Manuscript Received: April 29, 2009

\begin{abstract}
We use molecular dynamics simulations to study the influence of confinement on the dynamics of a nanoscopic water film at $T=300 \mathrm{~K}$ and $\rho=1.0 \mathrm{~g} \mathrm{~cm}^{-3}$. We consider two infinite hydrophilic ( $\beta$-cristobalite) silica surfaces separated by distances between 0.6 and $5.0 \mathrm{~nm}$. The width of the region characterized by surfacedominated slowing down of water rotational dynamics is $\sim 0.5 \mathrm{~nm}$, while the corresponding width for translational dynamics is $\sim 1.0 \mathrm{~nm}$. The different extent of perturbation undergone by the in-plane dynamic properties is evidence of rotational-translational decoupling. The local in-plane rotational relaxation time and translational diffusion coefficient collapse onto confinement-independent "master" profiles as long as the separation $d \geq 1.0 \mathrm{~nm}$. Long-time tails in the perpendicular component of the dipole moment autocorrelation function are indicative of anisotropic behavior in the rotational relaxation.
\end{abstract}

The properties of water confined in nanoscale geometries are of considerable scientific and technological interest. Nanoconfined water constitutes much of the environment in the cytoplasm of cells, where it acts as an active regulator of biological functions including protein folding. ${ }^{1}$ Understanding the transport and thermodynamic properties of water in molecular-scale geometries is key in a wide array of fields, from nanoand microfluidics, ${ }^{2}$ to cloud seeding in the atmosphere ${ }^{3}$ corrosion inhibition, ${ }^{4}$ and heterogeneous catalysis. ${ }^{5}$ Interesting phenomena arising in confined water have been observed experimentally, as well as in theoretical and computational studies. ${ }^{6-11}$ These include drastically different local structure and dynamics ${ }^{12,13}$ and the appearance of phase transitions not observable in the bulk under the same thermodynamic conditions. ${ }^{14}$ Such behavior arises from the subtle interplay of the fluid correlation and confinement geometric length scales ${ }^{15}$ and the nature of the interactions between water and the confining medium.

Recent experimental studies (e.g., see ref 16) have exploited the confinement of water in nanoscale geometries in order to investigate the possible existence of a liquid-liquid phase transition, terminating at a second critical point in supercooled water. Liquid-liquid coexistence has been postulated to occur in the deeply supercooled region of the phase diagram of water, at temperatures below the onset of homogeneous crystallization ( $231 \mathrm{~K}$ at 1 bar). ${ }^{17}$ By confining water in silica nanopores of 1.4-2.4 $\mathrm{nm}$ in diameter, Chen and co-workers were able to inhibit crystal nucleation and thereby study liquid water at temperatures down to $160 \mathrm{~K}$ (e.g., see refs 16, 18-22). Thus, confinement allows the study of a system under conditions that are inaccessible in the bulk. Because confining surfaces

* Towhom correspondence shouldbe addressed.E-mail:pdebene@ princeton. edu.

$\dagger$ Princeton University.

* Brooklyn College of the City University of New York. inevitably modify the structure and dynamics of proximal water, it is important to develop a fundamental understanding of the spatial range of such perturbations and, more generally, of the relationship between experiments performed on a confined system and its bulk behavior under otherwise identical conditions.

As a step in this direction, in this Letter we use molecular dynamics (MD) simulations to investigate the effect of the confinement length scale on the local and average dynamics in a nanoscale water film at ambient conditions. We do this by characterizing the regions in which different dynamical properties deviate from the bulk value under the influence of the confining surfaces. We consider a thin water film confined between two rigid, hydrophilic silica surfaces mimicking the chemistry of hydroxylated $\beta$-cristobalite. Periodic boundary conditions are imposed in the $x$ and $y$ directions, rendering the film macroscopic in the directions parallel to the surfaces. Confinement is imposed along the $z$-axis, normal to the surfaces, whose separation ( $d$, the confinement length scale) is varied between 0.6 and $5.0 \mathrm{~nm}$. We take $d$ as the distance between the planes containing the hydrogen atoms of each surface. Our MD simulations in the canonical ensemble are equilibrated at 300 $\mathrm{K}$ and a mean density of $1.0 \mathrm{~g} \mathrm{~cm}^{-3}$. Water-water interactions are modeled using the SPC/E pair potential, ${ }^{23}$ while water-surface interactions are treated using the potential by Lee and Rossky, ${ }^{10}$ which accounts for electrostatic and Lennard-Jones interactions between water and surface atoms. Further information about the system can be found in the Supporting Information and refs 11 and 24.

Our characterization is based on the local and average inplane translational diffusion coefficient and the in-plane rotational relaxation time (to be defined below). Figure 1a,b present the average dynamic properties, $D_{\|, \text {avg }}$ and $\tau_{\|, \text {avg }}$, as a function of $d$. The former is computed using the Einstein relation applied to the projection of the water $\mathrm{O}$-atom displacements onto a plane 


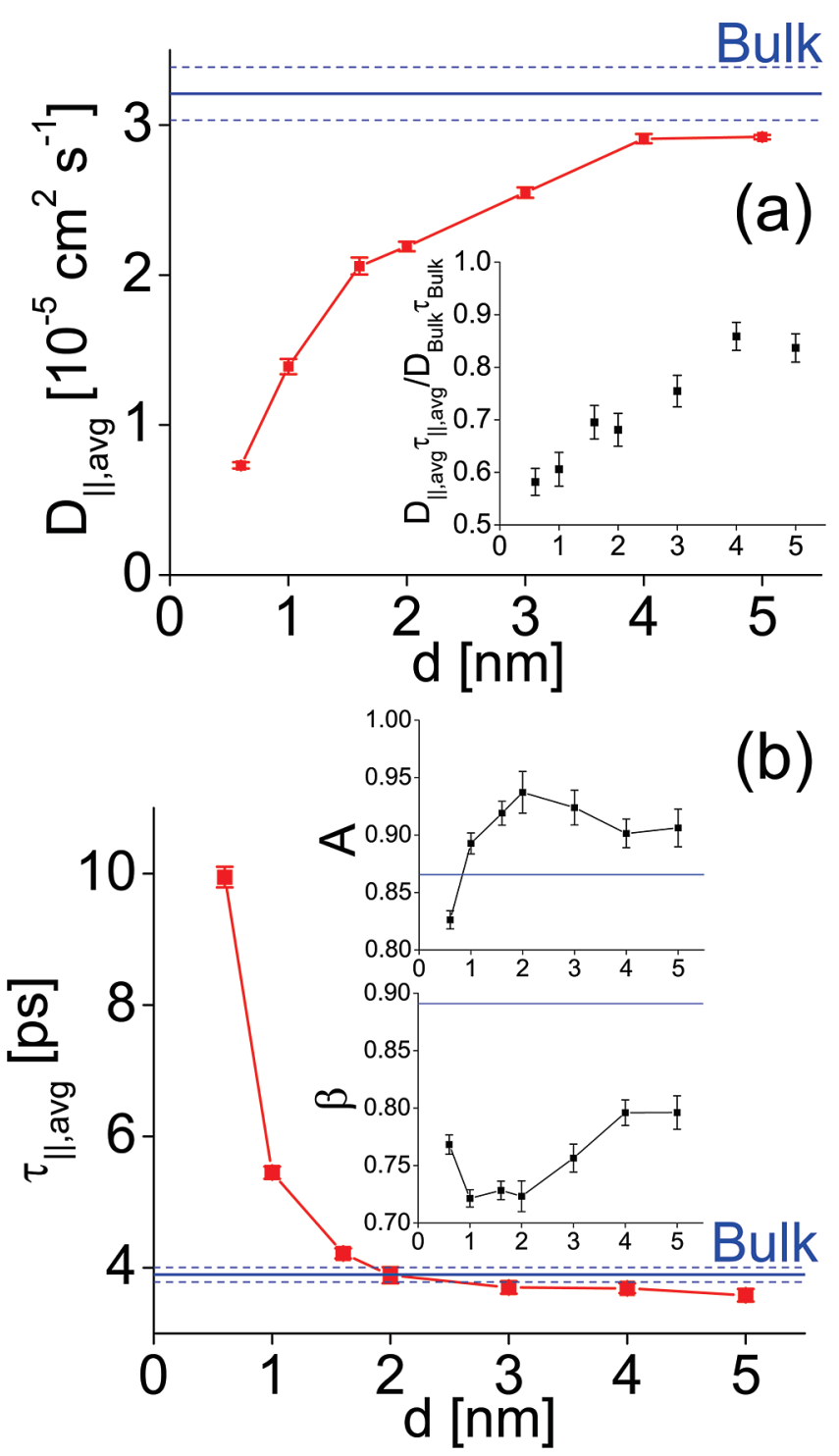

Figure 1. Average in-plane diffusion coefficient, $D_{\|, \text {avg }}$ (a), and rotational relaxation time, $\tau_{\|, \text {avg }}$ (b), as a function of confining surface separation, $d$. The inset in (a) presents the product of $D_{\|, \text {avg }}$ and $\tau_{\|, \text {avg }}$ normalized by the bulk properties as a function of $d$. Inset to (b): $d$ dependence of the $A$ and $\beta$ parameters obtained from stretched exponential fits. Error estimates for $D_{\text {Bulk }}$ and $\tau_{\text {Bulk }}$ are denoted by blue dashed lines. All data were obtained from MD simulations at $T=300$ $\mathrm{K}$ and $\rho_{\text {avg }}=1.0 \mathrm{~g} \mathrm{~cm}^{-3}$. The $x$-axes in the three insets are values of $d$ in $\mathrm{nm}$.

parallel to the surfaces $\left(\left\langle\Delta r_{\|}^{2}\right\rangle=4 D_{\|, \text {avg }} t\right.$, where $\Delta r_{\|}^{2}=\left(x-x_{0}\right)^{2}$ $\left.+\left(y-y_{0}\right)^{2}\right)$, while the latter is computed by fitting a stretched exponential function $\left(\varphi(t)=A \mathrm{e}^{-\left(t / \tau_{\|, a v g}\right)^{\beta}}{ }^{25}\right)$ to the dipole moment autocorrelation function $(\mathrm{ACF})$

$$
C_{\mathrm{Il,avg}}(t)=\left\langle\cos \theta_{\|}(t)\right\rangle
$$

We find that the decay of the dipole moment ACF is best described by a stretched exponential. This function has been used to model slow relaxation phenomena in supercooled, interfacial, and confined liquids. ${ }^{26-29}$ In eq $1, \theta_{\|}(t)$ is the angle formed by the in-plane projection of the water dipole moment unit vectors $\left(\vec{\mu}_{\|}=\mu_{x} \hat{\imath}+\mu_{x} \hat{\jmath}\right)$ at times 0 and $t$. The angular brackets denote averages over all molecules and starting times. Since all molecules, irrespective of their position relative to the surfaces, are considered in the ACF and in the Einstein relation, the resulting properties shown in Figure 1 reflect an average picture of particle mobility over regions exhibiting different dynamics.

Figure 1a shows that for all separations considered here, $D_{\|, \text {avg }}$ is smaller than $D_{\text {Bulk }}=3.21 \times 10^{-5} \mathrm{~cm}^{2} \mathrm{~s}^{-1}$ obtained from our simulations of bulk SPC/E water at $300 \mathrm{~K}$ and $1.0 \mathrm{~g} \mathrm{~cm}^{-3} \cdot{ }^{24}$ On the other hand, the average rotational mobility results presented in Figure $1 \mathrm{~b}$ show that $\tau_{\|, \text {avg }}$ reaches bulk-like values $\left(\tau_{\text {Bulk }}=3.89 \mathrm{ps}\right.$, obtained from our bulk SPC/E simulations at the same thermodynamic conditions) when $d \geq 2.0 \mathrm{~nm}$, while the $A$ and $\beta$ fitting constants, being larger and smaller, respectively, relative to those of the bulk, reflect slower rotational dynamics compared to the bulk. The inset to Figure 1a shows the product of $D_{\|, \text {avg }}$ and $\tau_{\|, \text {avg }}$, a quantity analogous to the ratio of $D_{\|, \text {avg }}$ to the rotational diffusion coefficient. It can be seen that this product is smaller than $D \tau_{\text {Bulk }}$ and increases with $d$, showing that the translational diffusion coefficient experiences a more pronounced decrease relative to its rotational counterpart as confinement becomes more severe. The inset to Figure 1a is therefore evidence of translational-rotational decoupling, a salient feature of supercooled liquids ${ }^{26}$ that has also been observed in nanoconfined water. ${ }^{30}$

A more informative picture of the impact of confinement on the dynamic properties of water is obtained from the local inplane diffusion coefficient and rotational relaxation time $\left(D_{\|}(z)\right.$ and $\left.\tau_{\|}(z)\right)$. We compute $D_{\|}(z)$ using a modified Einstein relation ${ }^{31}$ and $\tau_{\|}(z)$ through a local implementation of the dipole moment $\mathrm{ACF}$, analogous to the local mean-squared displacement (MSD) in ref 31 (cf. Supporting Information). In both of these implementations, the confined volume is discretized into thin slabs (thickness $=0.3 \mathrm{~nm}$ ), parallel to the surfaces, placed at various distances from the confining substrates. Only those molecules that are present in a given slab at the initial time and which remain within it for the entire observation time contribute to the computation of the local MSD or the ACF.

Figure 2a shows the local diffusion coefficient as a function of distance from the confining surfaces, $z$, for various $d$. We note that, to a good approximation, the data collapse onto a single curve when $d \geq 1.0 \mathrm{~nm}$. This finding suggests that, within this range of separations, in-plane local translational dynamics are independent of the confinement length scale. In addition, Figure $2 \mathrm{a}$ shows that the region of surface-dominated slowing down extends up to $\sim 1.0 \mathrm{~nm}$ away from the surfaces. The analysis in Figure 2a also presents evidence that the separationindependent diffusivity profile appears to break down under the smallest separation, $d=0.6 \mathrm{~nm}$.

The confinement-independent profiles in Figure $2 \mathrm{a}$ can be approximated by the function

$$
D_{\|}(z)=D_{\text {Bulk }}\left[1.0-\mathrm{e}^{-z / K_{\mathrm{D}}}\right]
$$

where $K_{\mathrm{D}}=0.46 \pm 0.03 \mathrm{~nm}$ is an adjustable parameter obtained from fitting eq 2 to the data with $d \geq 1.0 \mathrm{~nm}$. It is interesting to note that the range of $d$ for which the local diffusivity values collapse onto a "master" profile coincides with the range of separations where the density profiles also become independent of $d$ (inset to Figure 2a). In order to fully make the connection between local density and $D_{\|}(z)$, one should resolve the slabs in which the MSD is computed down to a thickness commensurate with that of slabs used in the calculation of the density profile (i.e., $\sim 0.04 \mathrm{~nm}$ ). The evaluation of MSD over such thin slabs (small sample size) would require much longer simulations. Nonetheless, a consistent picture emerges from Figure $2 \mathrm{a}$ and 

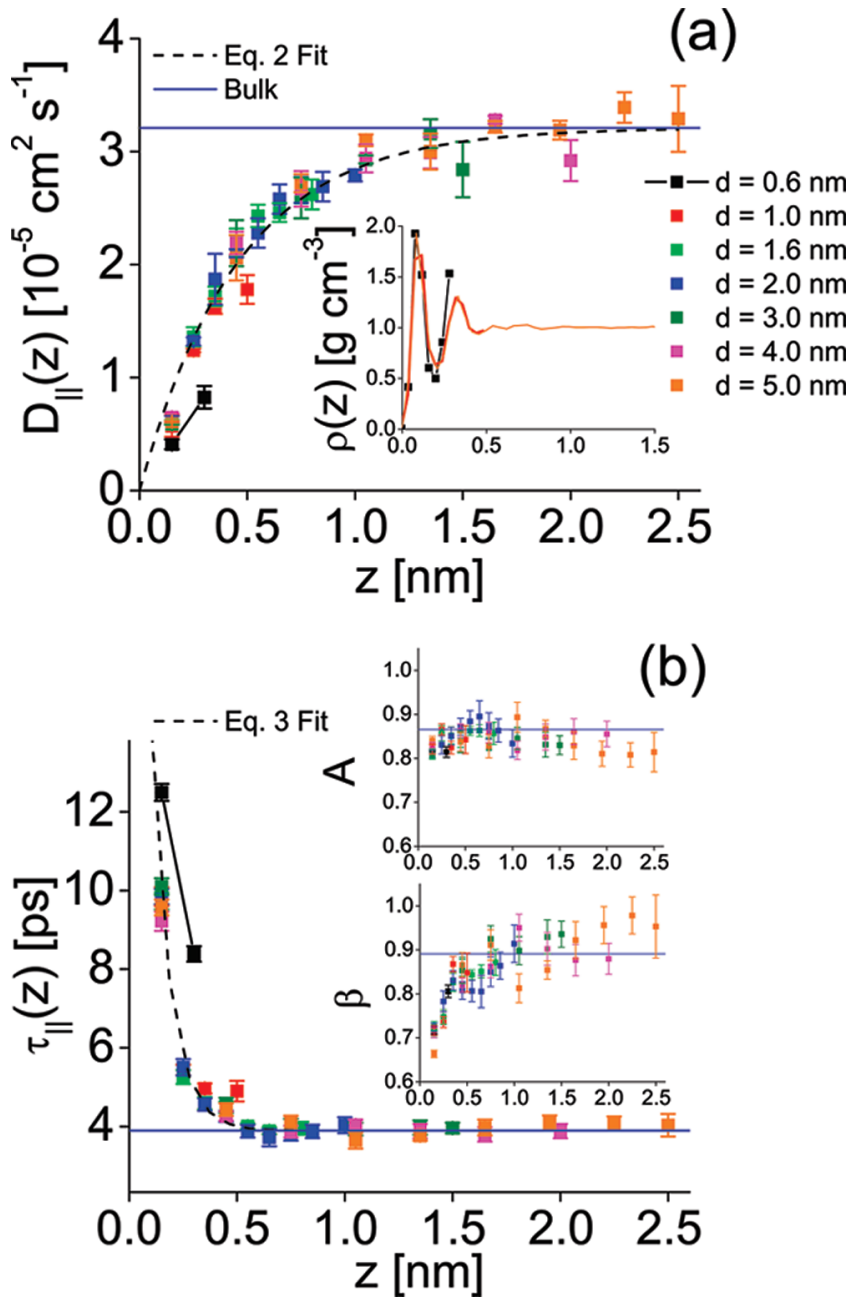

Figure 2. (a) Local in-plane diffusion coefficient of water, $D_{\|}(z)$, as a function of distance from the surfaces, $z$, for various surface separations, $d$. Inset to (a): local density, $\rho(z)$, computed in $0.041 \mathrm{~nm}$ thick slabs located at various distances from the surfaces (for clarity, only data for $d=0.6,1.0$, and $5.0 \mathrm{~nm}$ are shown). (b) Local in-plane rotational relaxation time, $\tau_{\|}(z)$, as a function of distance from the surfaces, $z$, for various surface separations, $d$. The $A$ and $\beta$ constants obtained from the stretched exponential fits are given in the insets to (b) as a function of $z$. The $x$-axes in the three insets are values of $z$ in $\mathrm{nm}$.

its inset insofar as the confinement-independent $D_{\|}(z)$ profile arises in systems with a hydration layer structure, as quantified by $\rho(z)$, that is also independent of $d$. Whether the $z$-independence of in-plane dynamics also holds in systems under hydrophobic (or mixed hydrophobic-hydrophilic) confinement or systems confined by flexible surfaces constitutes a direction for further study. We note recent studies by Mittal et al. ${ }^{32,33}$ on the confined hard-sphere fluid, which also highlight a one-toone correspondence between (static) equilibrium and dynamic properties. The authors obtained "master" profiles of $D_{\|, \text {avg }}$ versus excess entropy ${ }^{32}$ and the local $D$ perpendicular to the surfaces as a function of local insertion probability, ${ }^{33}$ over a broad range of conditions.

The local in-plane rotational relaxation time is presented in Figure $2 \mathrm{~b}$. The $\tau_{\|}(z)$ is calculated by fitting the decay of $C_{\|}(t)$ for $t \geq 1.0 \mathrm{ps}$ (computed through a local implementation of eq 1) to a stretched exponential function. We also report in the inset the $A$ and $\beta$ parameters extracted from the stretched exponential fits. Clearly, $\tau_{\|}(z)$ can also be described by a $d$ independent profile as long as $d \geq 1.0 \mathrm{~nm}$, which is well represented by the function

$$
\tau_{\text {|l }}(z)=\tau_{\mathrm{Bulk}}+K_{\tau, 1} \mathrm{e}^{-z / K_{\tau, 2}}
$$

where $K_{\tau, 1}=32 \pm 7$ ps and $K_{\tau, 2}=0.09 \pm 0.01 \mathrm{~nm}$ are obtained from fitting eq 3 to all data with $d \geq 1.0 \mathrm{~nm}$. Figure $2 \mathrm{~b}$ also shows that the region in which rotational dynamics are slowed down extends $\sim 0.5 \mathrm{~nm}$ away from the surfaces, that is, a region about one-half of that in which translational dynamics are perturbed relative to the bulk. This is further evidence of translational-rotational decoupling.

Much as in translational dynamics, the presence of confining surfaces results in anisotropic rotational motions ${ }^{34}$ since orientational randomization of a given vector fixed to the molecular reference frame (e.g., the $\mathrm{OH}$ vector of a water molecule or the dipole moment vector considered in this study) may be hindered by water-substrate interactions while being enhanced relative to the bulk when a different vector is chosen. ${ }^{35}$ Evidence of the anisotropic rotation of the dipole moment vector is shown in Figures $3 \mathrm{a}$ and $\mathrm{b}$, where we compare the decay of the in-plane average ACF of eq 1 (Figure 3a), from which we computed $\tau_{\|, \text {avg }}$ in Figure $1 b$, with that of the average out-of-plane ACF computed using the $z$-projection of $\vec{\mu}$, that is

$$
C_{z, \text { avg }}(t)=\frac{\left\langle\mu_{z}(0) \mu_{z}(t)\right\rangle}{\left\langle\mu_{z}(0) \mu_{z}(0)\right\rangle}
$$

Initially $(t<20 \mathrm{ps})$, the rate of decay of both ACFs in Figure 3 increases with $d$, as expected from the larger number of bulklike water molecules (i.e., those near the center of the confined volume) at larger $d$ values. Thereafter, two distinct observations are made; while this trend persists for $C_{\|, \text {avg }}(t)$, leading to its

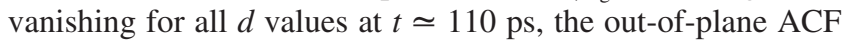
exhibits a tail indicative of long-time $\mu_{z}$ correlations. A similar plateau was observed in the MD simulations of Argyris et al. ${ }^{34}$ for the dipole moment ACF of water on high-density $\beta$-cristobalite. In previous work, ${ }^{36}$ we showed that water molecules adjacent to the surfaces orient themselves preferentially with both H-donor groups (and, consequently, the dipole moment) pointing toward the surfaces, thereby forming $\sim 2.7 \mathrm{HBs}$ with surface $\mathrm{OH}$ groups. ${ }^{24}$ The tail observed in $C_{z, \text { avg }}(t)$ is therefore due to water-surface $\mathrm{H}$-bonding, resulting in $\mu_{z}$ remaining weakly correlated for times exceeding 400 ps. The surprising observation in Figure $3 \mathrm{~b}$ is that the long-time correlation in $C_{z \text {,avg }}$ is not observed for the smallest separations investigated (the ACFs for $d=0.6$ and $1.0 \mathrm{~nm}$ vanish after $t \simeq 250 \mathrm{ps}$ ) but instead increases monotonically with separation up to $d=3.0$ $\mathrm{nm}$, at which point it appears to saturate. We attribute the absence of long-time correlations in $\mu_{z}$ when $d=0.6 \mathrm{~nm}$ to the partial breakdown of the HB network under severe confinement. We find evidence for this in the evolution of the surface-OH water $\mathrm{HB}$ autocorrelation function ${ }^{37}$ (see Supporting Information), noting that the surface water $\mathrm{HB}$ relaxation slows down as $d$ increases. This suggests that, as the surface separation increases, water molecules near the surfaces form a more stable HB network that hinders out-of-plane rotations. The progressive stabilization of the HB network is also manifested by the local number of $\mathrm{HBs}$ per water molecule, which exhibits a monotonic increase from $d=0.6$ up to $3.0 \mathrm{~nm}$, whereupon it appears to saturate (see Supporting Information). In experiments of water in nanoconfinement, ${ }^{38}$ the partial breakdown of the HB network explains why the viscosity of water remains within a factor of at most three relative to the bulk when confined by mica surfaces to films one or two monolayers in thickness. In our simulations, 

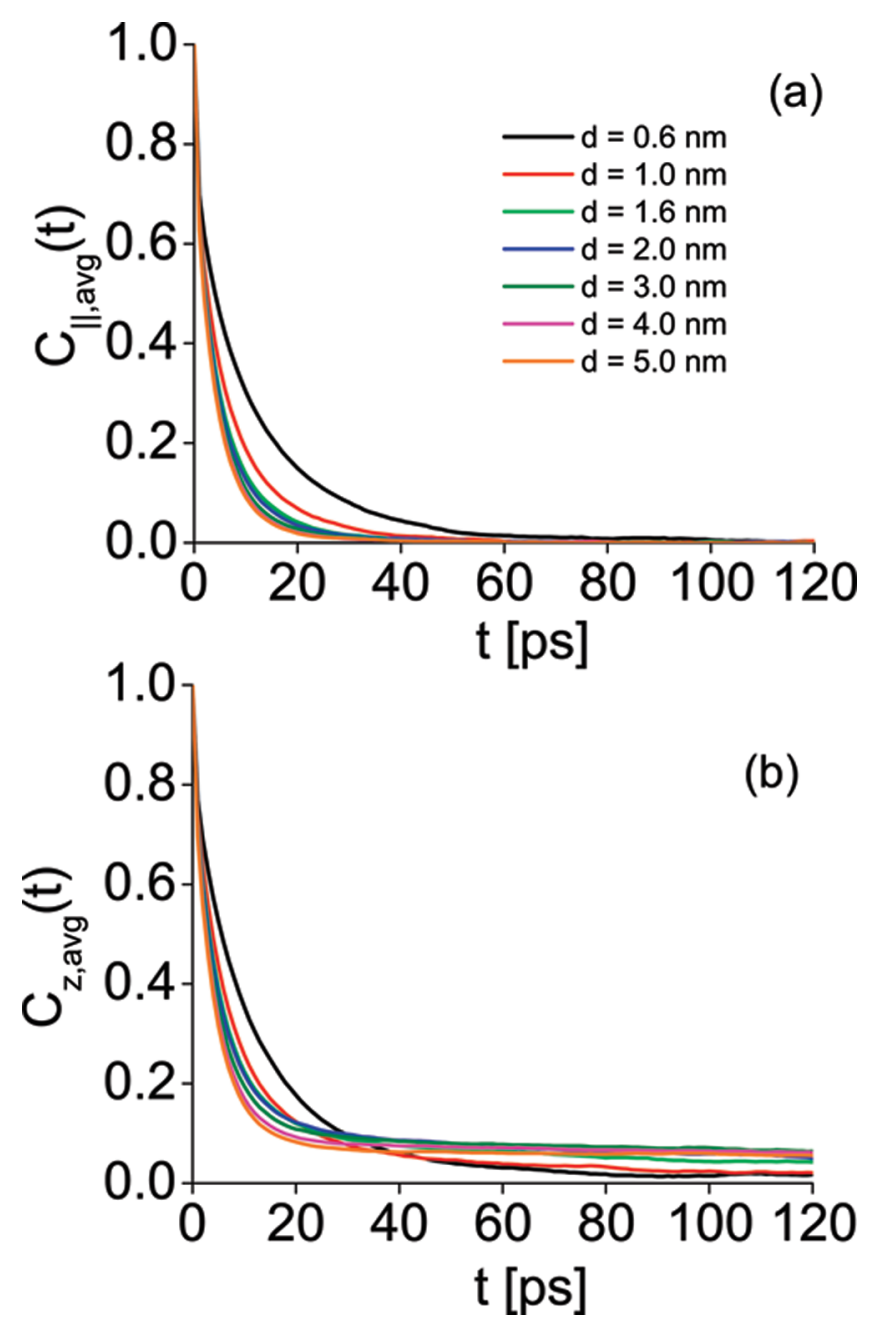

Figure 3. (a) Average in-plane dipole moment ACF, $C_{\|, \text {avg }}$ (b) Average out-of-plane dipole moment $\mathrm{ACF}, C_{z, \text { avg }}$.

the progressive stabilization of the HB network as $d$ increases gives rise to the long-time correlation in $C_{z, a v g}(t)$.

In summary, we have characterized the extent to which confinement affects the dynamic properties of a nanoscale water film. We have shown that regions of surface-dominated in-plane translational and rotational dynamics have different thickness and that the rotational relaxation time undergoes a smaller perturbation compared to the translational diffusion coefficient. Our most important observation is the existence of confinementindependent profiles for the in-plane dynamic properties when $d>0.6 \mathrm{~nm}$.

Acknowledgment. Financial support for this work was provided by MRSEC NSF (DMR-0213706) and ARO-MURI (W911NF-04-1-0170). S.R.-V.C. acknowledges useful discussions with T. G. Lombardo.

Supporting Information Available: Further information on our MD simulations, hydrogen bonding, and the calculation of dynamic properties. This material is available free of charge via the Internet at http://pubs.acs.org.

\section{References and Notes}

(1) Ball, P. Chem. Rev. 2008, 108 (1), 74-108.

(2) Stone, H. A.; Stroock, A. D.; Ajdari, A. Annu. Rev. Fluid Mech. 2004, 36, 381-411.
(3) Shevkunov, S. V. Colloid J. 2005, 67 (4), 497-508.

(4) Marcus, P., Ed. Corrosion Mechanisms in Theory and Practice, 2nd ed.; Marcel Dekker: New York, 2002.

(5) Goettmann, F.; Sanchez, C. J. Mater. Chem. 2007, 17 (1), 24-30.

(6) Moilanen, D. E.; Levinger, N. E.; Spry, D. B.; Fayer, M. D. J. Am. Chem. Soc. 2007, 129 (46), 14311-14318.

(7) Gallo, P.; Rovere, M. J. Phys.: Condens. Matter 2003, 15 (45), $7625-7633$.

(8) Thompson, H.; Soper, A. K.; Ricci, M. A.; Bruni, F.; Skipper, N. T. J. Phys. Chem. B 2007, 111 (20), 5610-5620.

(9) Truskett, T. M.; Debenedetti, P. G.; Torquato, S. J. Chem. Phys. 2001, 114 (5), 2401-2418.

(10) Lee, S. H.; Rossky, P. J. J. Chem. Phys. 1994, 100 (4), 33343345.

(11) Giovambattista, N.; Rossky, P. J.; Debenedetti, P. G. Phys. Rev. E 2006, 73 (4), 041604.

(12) Gallo, P. Rovere, M. Spohr, E. Phys. Rev. Lett. 2000, 85 (20), $4317-$ 4320. This early work on water dynamics inside of a cylindrical silica nanopore showed that molecules within $\sim 0.5 \mathrm{~nm}$ of the pore surface behave as glassy water, even at room temperature, while water near the pore center is bulk-like.

(13) Gallo, P.; Ricci, M. A.; Rovere, M. J. Chem. Phys. 2002, 116 (1), 342-346.

(14) Giovambattista, N.; Rossky, P. J.; Debenedetti, P. G. Phys. Rev. Lett. 2009, 102 (5), 050603.

(15) Klapp, S., Schoen, M., Eds. Reviews in Computational Chemistry; John Wiley \& Sons: Hoboken, NJ, 2007; Vol. 24,.

(16) Liu, L.; Chen, S. H.; Faraone, A.; Yen, C. W.; Mou, C. Y. Phys. Rev. Lett. 2005, 95 (11), 117802.

(17) Debenedetti, P. G.; Stanley, H. E. Phys. Today 2003, 56 (6), 4046.

(18) Zanotti, J. M.; Bellissent-Funel, M. C.; Chen, S. H.; Kolesnikov, A. I. J. Phys.: Condens. Matter 2006, 18 (36), S2299-S2304.

(19) Liu, L.; Chen, S. H.; Faraone, A.; Yen, C. W.; Mou, C. Y.; Kolesnikov, A. I.; Mamontov, E.; Leao, J. J. Phys.: Condens. Matter 2006, 18 (36), S2261-S2284.

(20) Mallamace, F.; Broccio, M.; Corsaro, C.; Faraone, A.; Wanderlingh, U.; Liu, L.; Mou, C. Y.; Chen, S. H. J. Chem. Phys. 2006, 124 (16), 161102.

(21) Chen, S. H.; Mallamace, F.; Mou, C. Y.; Broccio, M.; Corsaro, C.; Faraone, A.; Liu, L. Proc. Natl. Acad. Sci. U.S.A. 2006, 103 (35), 12974 12978.

(22) Mallamace, F.; Broccio, M.; Corsaro, C.; Faraone, A.; Majolino, D.; Venuti, V.; Liu, L.; Mou, C. Y.; Chen, S. H. Proc. Natl. Acad. Sci. U.S.A. 2007, 104 (2), 424-428.

(23) Berendsen, H. J. C.; Grigera, J. R.; Straatsma, T. P. J. Phys. Chem. 1987, 91 (24), 6269-6271.

(24) Romero-Vargas Castrillón, S.; Giovambattista, N.; Aksay, I. A.; Debenedetti, P. G. J. Phys. Chem. B 2009, 113, 1438-1446. Our $D_{\text {Bulk }}$ is $28 \%$ higher than the literature value for SPC/E water, $2.5 \times 10^{-5} \mathrm{~cm}^{2} \mathrm{~s}^{-1} .23$ This discrepancy is due to the different system sizes and methods of truncation for electrostatic interactions used in ref 23 and our work.

(25) Williams, G.; Watts, D. C. Trans. Faraday Soc. 1970, 66 (565P), $80-85$.

(26) Debenedetti, P. G.; Stillinger, F. H. Nature 2001, 410 (6825), 259267.

(27) Kumar, P.; Franzese, G.; Buldyrev, S. V.; Stanley, H. E. Phys. Rev. E 2006, 73 (4), 041505.

(28) Oleinikova, A.; Smolin, N.; Brovchenko, I. Biophys. J. 2007, 93 (9), 2986-3000.

(29) Faraone, A.; Liu, L.; Mou, C. Y.; Shih, P. C.; Copley, J. R. D.; Chen, S. H. J. Chem. Phys. 2003, 119 (7), 3963-3971.

(30) Harpham, M. R.; Ladanyi, B. M.; Levinger, N. E.; Herwig, K. W. J. Chem. Phys. 2004, 121 (16), 7855-7868.

(31) Liu, P.; Harder, E.; Berne, B. J. J. Phys. Chem. B 2004, 108 (21), 6595-6602.

(32) Mittal, J.; Errington, J. R.; Truskett, T. M. Phys. Rev. Lett. 2006, 96 (17), 177804.

(33) Mittal, J.; Truskett, T. M.; Errington, J. R.; Hummer, G. Phys. Rev. Lett. 2008, 100 (14), 145901.

(34) Argyris, D.; Tummala, N. R.; Striolo, A.; Cole, D. R. J. Phys. Chem. C 2008, 112 (35), 13587-13599.

(35) Mukherjee, B.; Maiti, P. K.; Dasgupta, C.; Sood, A. K. Acs Nano 2008, 2 (6), 1189-1196.

(36) Giovambattista, N.; Debenedetti, P. G.; Rossky, P. J. J. Phys. Chem. B 2007, 111 (32), 9581-9587.

(37) Liu, P.; Harder, E.; Berne, B. J. J. Phys. Chem. B 2005, 109 (7), 2949-2955.

(38) Raviv, U.; Laurat, P.; Klein, J. Nature 2001, 413 (6851), 51-54.

JP9025392 